\title{
Spinal Cord management. Looking into attitude and knowledge of caregivers and patients
}

\author{
JJ Wyndaele, Editor \\ Antwerp University Hospital, Antwerp, Belgium \\ E-mail: spinalcord@uza.be
}

Dear Spinal Cord reader,

It is with great satisfaction that we can announce that the 2008 Impact factor for Spinal Cord, released in June 2009, has increased to $>2$. This reflects, for example, the ever increasing quality of the manuscripts accepted for publication and the great work done by our reviewers. We will do our utmost best to continue in the same line.

In this issue again, we present very important work. An international expert consensus statement by Guest et al. addresses the ethical problems created by the sale of unproven cellular therapies to patients with spinal cord injury.

The review by Gelis et al. discusses the pressure ulcer risk factors in persons with spinal cord injury during the chronic stage. Behavioural risk factors (relieving the pressure, careful skin monitoring and smoking) are probably the ones for which a preventive strategy can be established. It is important to develop specific assessment tools for these behavioural risk factors in order to determine their relevance and evaluate the impact of therapeutic educational programs. A previous contribution by the same group reviewed the literature on ulcer risk in the acute and rehabilitation stage (Spinal Cord 2009 Feb;47(2):99-107) and found that there is insufficient evidence to make a recommendation on medical risk factors, except for low blood pressure upon admission to the Emergency Room, with a moderate level of evidence. Regarding the rehabilitation stage, no study was deemed relevant.

Krause and Saunders et al. assessed the risk factors associated with hospitalization and the relationship of individual health behaviours with hospitalizations following spinal cord injury (SCI). Two biographic and injury characteristics were associated with hospitalization: race and SCI severity. Specifically, minorities and persons with non-motor functional high cervical or non-cervical SCI (ASIA grades A-C) were more likely to be hospitalized. Three behavioural factors were significantly associated with hospitalization: persons who used prescription medications, those who engaged more in smoking behaviours, and persons who reported more SCI specific health behaviours were more likely to be hospitalized.

A questionnaire-based survey by Furlan et al. identified significant differences in the attitudes toward elderly patients between nurses working in the acute care setting versus rehabilitation setting who showed similar attitudes towards SCI individuals. The most reliable factor associated with the nurses' attitudes was their level of education.

Schottler et al. describe the prevalence and knowledge of autonomic dysreflexia (AD) from the perspectives of patients - between 1 and 21 years old - and caregivers. The study evaluated the answers to the important questions: Does the patient experience AD? Does the patient/caregiver know what $\mathrm{AD}$ is? Can the patient/caregiver name three signs/symptoms of an $\mathrm{AD}$ episode. Does the patient/caregiver know how to treat AD?

A study by Vogel et al. reports the intra-rater reliability of anorectal sensory and motor examinations and classification of injury severity in children with SCI. The study does not fully support the use of anorectal examinations as a pediatric measure for clinical use or for SCI clinical trials when involving children. The inadequate reliability of the anorectal examinations in this study sample suggests that further research is needed.

Several more very interesting studies can also be found in this issue.

Enjoy exploring them

Spinal Cord (2009) 47, 649; doi:10.1038/sc.2009.110 